\title{
svaRetro and svaNUMT: Modular packages for annotation of retrotransposed transcripts and nuclear integration of mitochondrial DNA
} in genome sequencing data

\section{Authors:}

Ruining Dong ${ }^{1,2}$, Daniel Cameron ${ }^{1,2,4}$, Justin Bedo ${ }^{1,3, *}$, Anthony T Papenfuss ${ }^{1,2,4,5, *, * *}$

\section{Affiliations:}

${ }^{1}$ Bioinformatics Division, Walter and Eliza Hall Institute of Medical Research, Parkville, VIC 3052, Australia.

${ }^{2}$ Department of Medical Biology, University of Melbourne, VIC 3010, Australia.

${ }^{3}$ School of Computing and Information Systems, University of Melbourne, VIC 3010, Australia.

${ }^{4}$ Peter MacCallum Cancer Centre, Melbourne, VIC 3000, Australia.

${ }^{5}$ Sir Peter MacCallum Department of Oncology, University of Melbourne, VIC 3010, Australia.

*These authors contributed equally.

**To whom correspondence should be addressed: Anthony $\mathrm{T}$ Papenfuss (papenfuss@wehi.edu.au) 


\begin{abstract}
Background

The biological significance of structural variation is now more widely recognized. However, due to the lack of available tools for downstream analysis, including processing and annotating, interpretation of structural variant calls remains a challenge.
\end{abstract}

\title{
Findings
}

Here we present svaRetro and svaNUMT, R packages that provide functions for annotating novel genomic events such as non-reference retro-copied transcripts and nuclear integration of mitochondrial DNA. We evaluate the performance of these packages to detect events using simulations and public benchmarking datasets, and annotate processed transcripts in a public structural variant database.

\section{Conclusions}

svaRetro and svaNUMT provide efficient, modular tools for downstream identification and annotation of structural variant calls.

Keywords: structural variants; retrotransposed transcripts; nuclear-mitochondrial fusions. 


\section{Findings}

\section{Background}

Structural variants (SVs) are polymorphisms and mutations commonly observed in the genome. They range from simple insertions and deletions to complex chromosomal-scale rearrangements. SVs are a significant source of genomic variability in humans, and SV analysis has rapidly become a part of standard pipelines in genomic studies [1-4]. To call SVs from short-read DNA sequencing data derived from individual samples (e.g., germline or cell lines), matched tumour-normal pairs, or multiple related samples, a variety of tools have been developed [5-7].

Interpretation of SV calls requires additional downstream analyses. For example, in tumour genome analysis, annotation of genes disrupted by SVs is a relatively straightforward downstream analysis; due to the splicing logic required, fusion gene prediction is more complicated (e.g. LINX [8]); while inference of chromothripsis [9] or chromoplexy [10]. There are relatively few modular tools available for downstream annotation of SVs. Further complicating this issue, some SV annotation software is highly dependent on specific SV callers (e.g. LINX with GRIDSS) or completely integrated (e.g. AmpliconArchitect [11] and RetroSeq [12]). To cope with the ever-growing number of SV datasets downstream of calling SVs, users need high-quality tools to annotate and understand these calls. Two biological phenomena that are currently underserved by available tools are Nuclear Mitochondrial insertion (NUMTs) [13] and retroposed transcript (RT) insertion.

Nuclear mitochondrial integrations (NUMTs) are formed during mitosis when the nuclear membrane breaks down, allowing mitochondrial DNA (mtDNA) to escape from degrading mitochondria, which is accelerated in cancer, and migrate into the nuclear genome $[14,15]$. 
NUMTs are present in the normal genome, having integrated during evolution. Somatic NUMT events have been observed in human cancer cells, but have not been extensively studied, and further investigation is needed to understand their extent and role in cancer development [16,17]. Despite their potential biological significance, these events are often overlooked [18].

Retroposed transcripts (RTs) are associated with LINE element reactivation in cancer [19] but also occur in the germline leading to processed pseudogenes [20]. RTs can interfere with the expression of their parent genes [21], generate antisense transcripts [22], and compete for microRNA binding with their parent genes [23]. Additionally, mutations introduced by the process may drive cancer evolution, particularly when the retroposed transcript is inserted into another gene.

Here, we present two R packages for the downstream analysis of SVs. svaNUMT and svaRetro provide flexible frameworks to analyze and explore NUMTs and RTs. In typical use, SV calls in a VCF file are loaded into a breakend-centric GRanges object using VariantAnnotation [24] and StructuralVariantAnnotation [25]. The packages then search for evidence supporting events of interest. For RT detection, svaRetro also requires a $\mathrm{TxDb}$ object which stores transcript metadata. The TxDb object can be loaded via pre-existing annotation packages or generated from existing data [26]. The output of the packages are lists of GRanges objects that can be converted to various data formats, including BEDPE, supporting further analysis (Figure 1). 


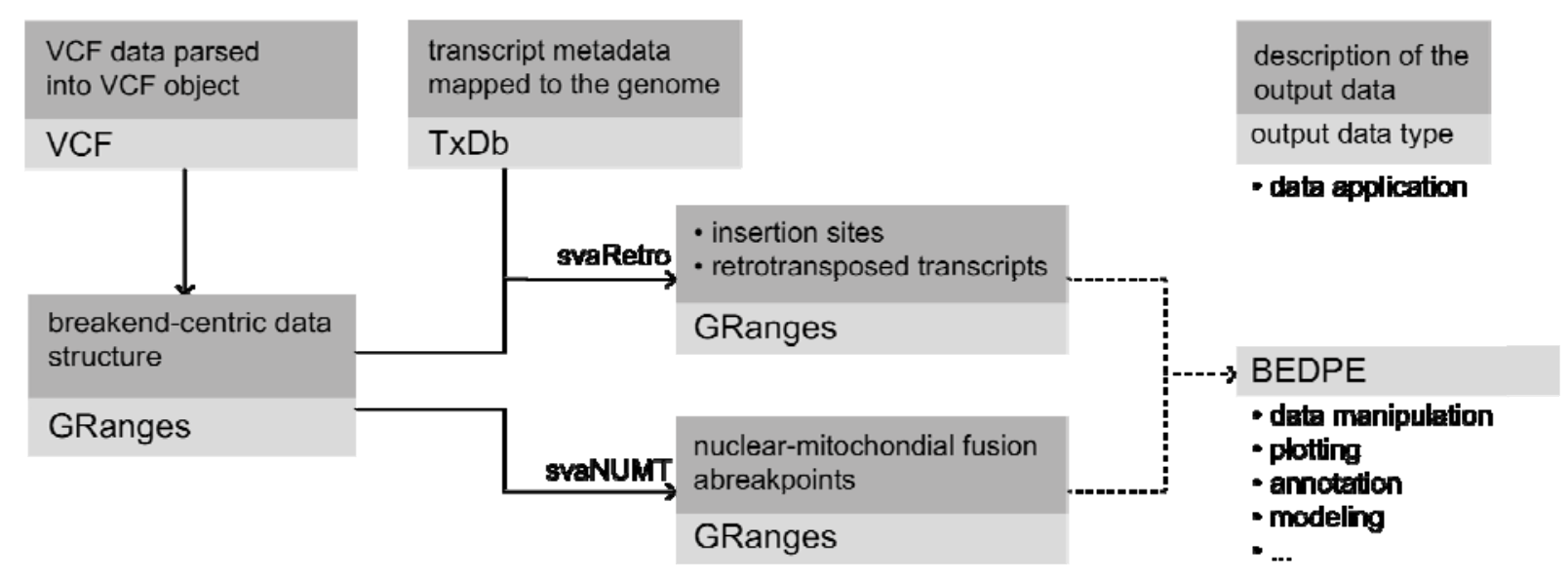

Figure 1. Workflow of svaRetro and svaNUMT. SV calls are first loaded as VCF objects with

VariantAnnotation [24], then converted into breakend-centric GRanges with

StructuralVariantAnnotation [25]. svaRetro takes as input the Granges data and a TxDb

annotation object, which stores the transcript metadata. The output of svaRetro is a list of

GRanges grouped by the source gene of the retrotransposed transcripts. svaNUMT requires

only the GRanges object as input. The results are grouped by events and the locations of the

breakends. The output can be easily converted to BEDPE format, which is commonly used

for downstream analyses.

Input and Output Data Format

Both svaRetro and svaNUMT take as input a GRanges object with a breakend-centric notation, where a GRanges record is used to represent each breakend, and a breakpoint consists of a pair of breakends. Although breakpoint-centric data structures are available for SV representation (e.g., Pairs object in rtracklayer [27]), we have chosen the breakendcentric notation as it simplifies frequent operations in the analysis such as overlap finding with genes and repeats. 
The output formats of svaRetro and svaNUMT are GRanges to support flexible downstream analyses. In cases where alternative formats are required, StructuralVariantAnnotation [25] provides functions for format conversion between BEDPE and Pairs [28] with GRanges.

\section{Identifying Retrotransposed Transcripts}

SvaRetro identifies RTs using the provided SV calls. RTs are processed transcripts integrated into the genome and characterized by intronic losses and polyadenylation. The candidate insertion sites are scattered across the genome due to the mobilization of transposable elements and are frequently combined with target site duplications (TSD). Therefore, except when the transcript comprises only a single exon, an RT should show a signature of intronic deletions-breakpoints aligned with adjacent exon boundaries from the same mRNA transcript. Additionally, the insertion site is detectable as a rearrangement connecting an exonic edge and a second genomic location (see Figure 2). 
(a)

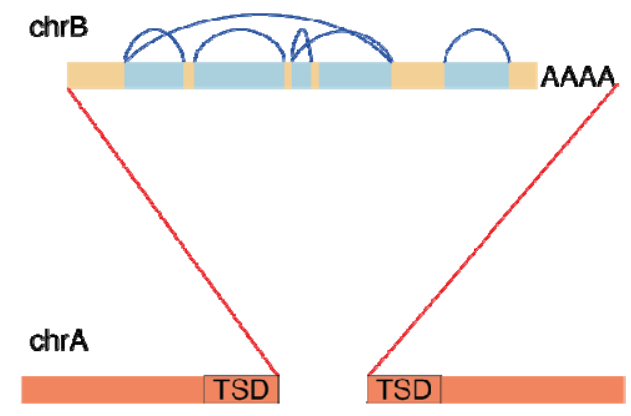

(b)

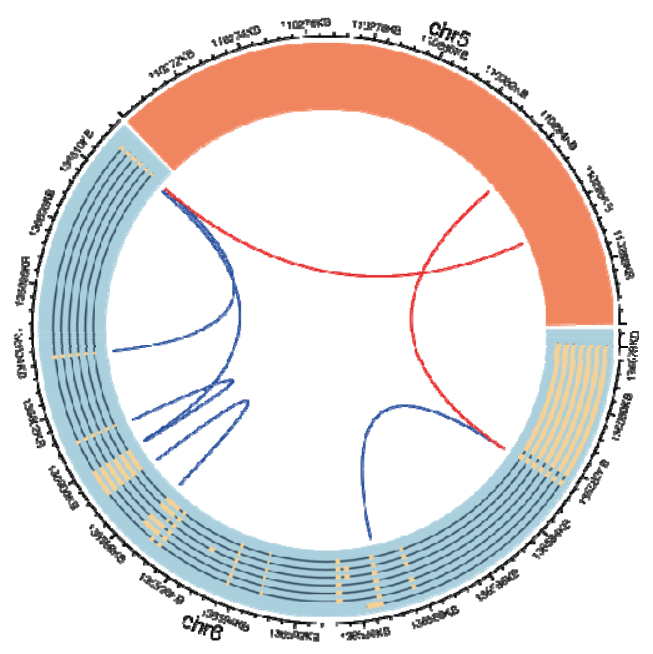

Insertion site

exon-exon junction

Figure 2. Breakpoint signatures of RT events. (a) A multi-exon RT consists of two breakpoint signatures: exon-exon junctions (blue) and fusions of exon and insertion sites (red). Polyadenylation and target site duplication (TSD) may also be present. (b) A germline BCLAF1 RT on chr5 detected in sample COLO829 [29] with breakpoint calls of exon-exon junctions (blue) and insertion sites (red). Not all exon-exon junctions were detected by the SV caller, and a transitive call connecting exon 1 and exon 4 is also evident.

To detect intronic deletions, overlaps between breakend positions and exon-intron (and intron-exon) boundaries are returned under a maximum gap threshold (default of $100 \mathrm{bps}$ ). An RT is reported with higher confidence when more exons are present. This quality, denoted by minscore, is evaluated using the proportion of intronic deletions detected from the total possible in the transcript. Depending on the resolution of the SV caller, small exons (e.g. shorter than the read length) can be missed or captured in transitive breakpoints (i.e. a pair of adjacent rearranged segments A-to-B and B-to-C are detected as A-to-C). Meanwhile, breakpoints consisting of an exon boundary and a second genomic location are potential 
insertion sites. Due to the frequent 5 ' truncation in retrotransposons, the maximum gap threshold does not apply here.

The output is a list of GRanges objects consisting of breakend-centric SV calls grouped by the source gene of the retroposed transcript. Each grouped event contains candidate insertion sites and exon-exon junctions, if available. Each candidate insertion site is annotated by the potential source transcript(s) and whether exon-exon junctions are detected for the source transcript(s). Exon-exon junction calls are annotated by the exon indices, corresponding transcripts satisfying the minscore threshold, and NCBI gene symbols.

RT insertion sites can be discovered on both 5' and 3' sides, only one side, or none. An insertion site could be missed, even when the breakend is reported in the SV callset, due to a sizable 5' truncation despite the tolerant threshold, a 5' inversion, or a combination of rearrangements.

\section{Identifying Nuclear-mitochondrial Genome Fusion Events}

svaNUMT searches for NUMT events by identifying SVs (in breakend notation) supporting the fusion of nuclear chromosome and mitochondrial genome. In the event of mtDNA integration in nuclear genomes, it is expected that split reads and discordant reads are detected near the integration sites. These features, when picked up by a structural variant caller, are represented as translocation events between mtDNA and nuclear DNA in the SV calls, given the mitochondrial reference genome is included in the library (see Figure 3). 
A NUMT event consists of two insertion sites, which can be linked by phasing nearby events. svaNUMT annotates linked insertion sites where possible. Candidate linked nuclear insertion sites are reported by events as a list of GRanges.

(a)

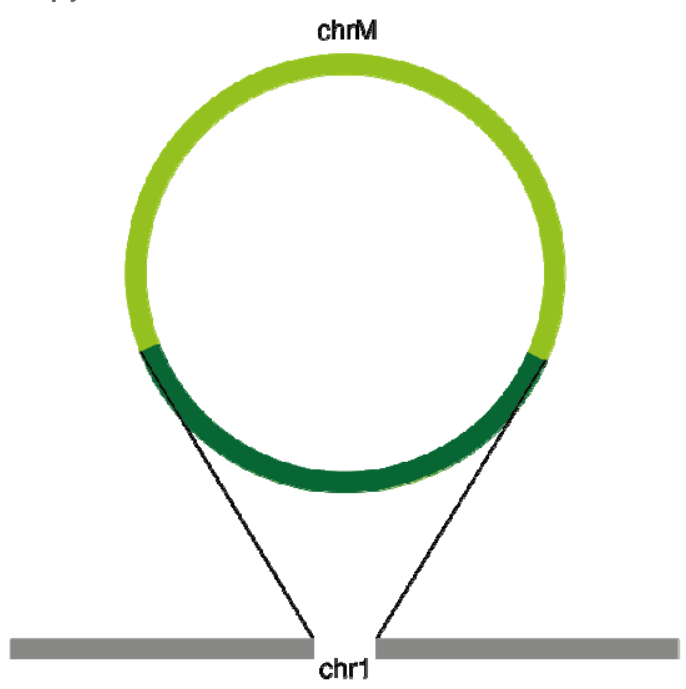

(b)

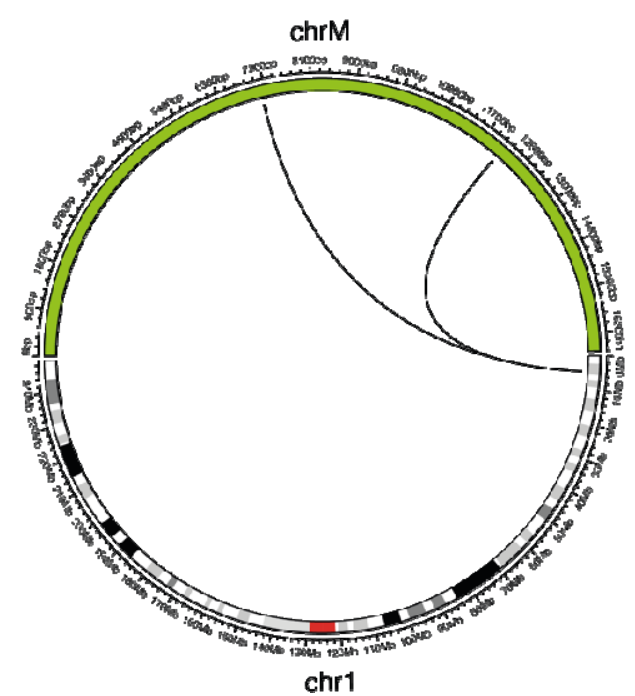

Figure 3. Breakpoint signatures of NUMTs. (a) Schematic of a NUMT event where a sequence from chrM (dark green) is inserted into a nuclear chromosome (chrA). The sequencing read features consists of one breakpoint connecting chrA and chrM for each insertion site. (b) A NUMT event detected in chrl of the NUMT simulation (described in the text).

\section{Benchmarking and Application}

\section{Testing on simulated data}

We next tested svaRetro and svaNUMT using 500 non-overlapping simulated events on chromosome 1. To generate these, chromosome 1 was first divided into 570 uniform intervals. Of these, 507 overlapped (at least $80 \%$ overlap) the set of high-confidence Tier 1 regions defined by Zook et al [30]. Intervals not in high confidence regions were excluded. A final set of 500 intervals was then randomly selected. A different random transcript sequence, 
accompanied by a polyadenylation sequence, was inserted into a random location of each interval. Simulated NUMTs were generated through insertions of $500 \mathrm{mtDNA}$ sequences with polyadenylation on the chr1 sequence, where insertion sites were selected using the same method as described above. The mtDNA sequences included 50 each of lengths 10, 20, $50,100,200,500,1,000,2,000,5,000$, and 10,000 base pairs (bp). Paired-end reads at 30x mean coverage were simulated using Art [31] using the HiSeq 2500 error profile for both simulated RTs and simulated NUMTs. SV breakpoints of both samples were called by Manta [6] and GRIDSS [5]. We then used svaRetro and svaNUMT to detect simulated events and analyzed the results with manual inspection.

svaRetro detected 470 out of 500 (94\%) RTs from the GRIDSS calls and 443 out of 500 (86\%) from Manta calls (candidateSV callset). 23 of the 30 undetected events did not have a breakpoint called by GRIDSS within 100bp of the insertion sites. Out of the seven events where breakends were detected near the insertion sites, 5 events were not mapped to the reference genome, 1 event had secondary calls mapped to an alternative locus, and 1 event was mapped to alternate assembly. Of the 57 undetected events from the Manta calls, 45 did not have a breakpoint called within 100bp of the insertion sites, 11 insertion sequences were not mapped to the reference, and 1 inserted transcript was mapped to an alternate assembly.

svaNUMT detected 276 NUMTs from GRIDSS SV calls and 202 from Manta SV calls. Among the undetected NUMT events, some were due to undetected breakpoints near the insertion sites by the SV callers (14 from GRIDSS and 75 from Manta); some were due to unmapped insertion sequences (122 from GRIDSS and 105 from Manta), where the majority were 10 and $20 \mathrm{bp}$ events. In the remainder of the events, only one insertion site breakpoint 
detected reported (88 from GRIDSS and 118 from Manta). The summary of the results is shown in Figure 4.

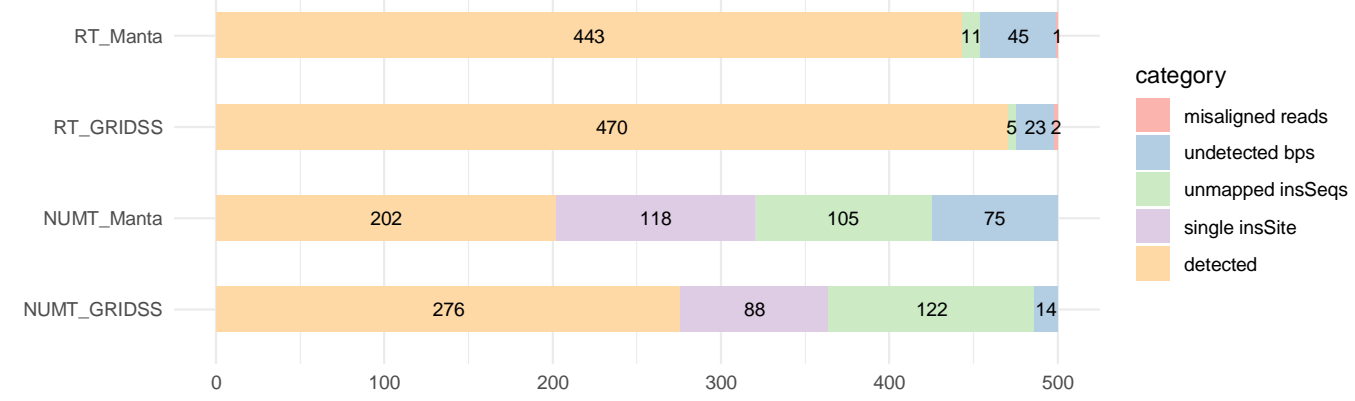

Figure 4 Detection results of the 500 simulated RT and NUMT events by svaRetro and svaNUMT using SV callsets produced by Manta and GRIDSS. For simulated RTs, the undetected events fell into one of categories of unmapped insertion sequences (insSeqs), undetected breakpoints (bps), or misaligned reads. For simulated NUMTs, the unreported events either had one insertion site breakpoint undetected (single insSite), unmapped insSeqs, or undetected bps.

\section{Benchmarking against existing tool}

While there were tools developed for transposable elements from WGS data, the detection of RTs has not been addressed. To our best knowledge, GRIPper is the only publicly available RT detection tool [32]. We applied svaRetro and GRIPper on human germline and tumour datasets, namely HG002, a GIAB cell line using 60× coverage WGS [33], and COLO829, a tumour cell line derived from a cutaneous melanoma [29] with matched lymphoblastoid (normal) cell line. For svaRetro, we used GRIDSS to call SVs on these samples. The results were compared with manual inspection. To our knowledge, no publicly available tools exist to detect NUMTs. Consequently, we were unable to benchmark svaNUMT. 
HG002: GRIPper reports four instances of RTs in HG002, all of which are detected by svaRetro under the same matching threshold of GRIPper. In addition, svaRetro reports the exon-exon junctions and trace the source of the insertion sequence to the specific transcripts, which is absent in GRIPper (Supplementary File 1).

COLO829: Three instances of RTs were detected by GRIPper in both tumour and matched normal samples. 1 instance of RT is detected in normal sample only (Supplementary File 2 and 3). For one event detected by GRIPper in both the tumour and the normal samples, the inserted transcript sequence mapped to a processed pseudogene (on chr1) as well as the source gene (on chr16). GRIDSS reported this event as a translocation of the in-reference pseudogene from chr1, therefore this event was not reported by svaRetro (see Supplementary Figure 1). The rest RT events were successfully identified by svaRetro.

The discrepancy is the result of breakpoint evidence required for RT discovery. GRIPper reports putative insertion sites using only split reads, while svaRetro collects information on both exon - exon junctions and candidate RT insertion sites, based on breakpoints detected by the structural variant caller of choice.

\section{Application to gnomAD-SV database}

We established a catalogue of non-reference RTs using svaRetro on the gnomad-SV dataset [34], where RTs were largely unannotated. NUMT annotation was not applicable as mitochondrial SVs were excluded from the database. In total, 53,529 candidate insertion sites were detected by svaRetro, including events single-exon transcript insertions and/or with insertions with only one side of the insertion detected. The distribution of all source genes and candidate insertion sites are shown in Figure 5. 1298 high-confidence insertion sites were 
supported by exon-exon junctions and high-quality SV breakpoint calls (using the "PASS"

filter). RT insertions can be detected in non-repetitive sequence and across different types of

repetitive sequence (Figure 6).

chr1

chr2

chr3

chr4

chr5

chr6

chr7

chr8

chr9

chr10

chr11

chr12

chr13

chr14

chr15

chr16

chr17

chr18

chr19

chr20

chr21

chr22

chrX

chrY

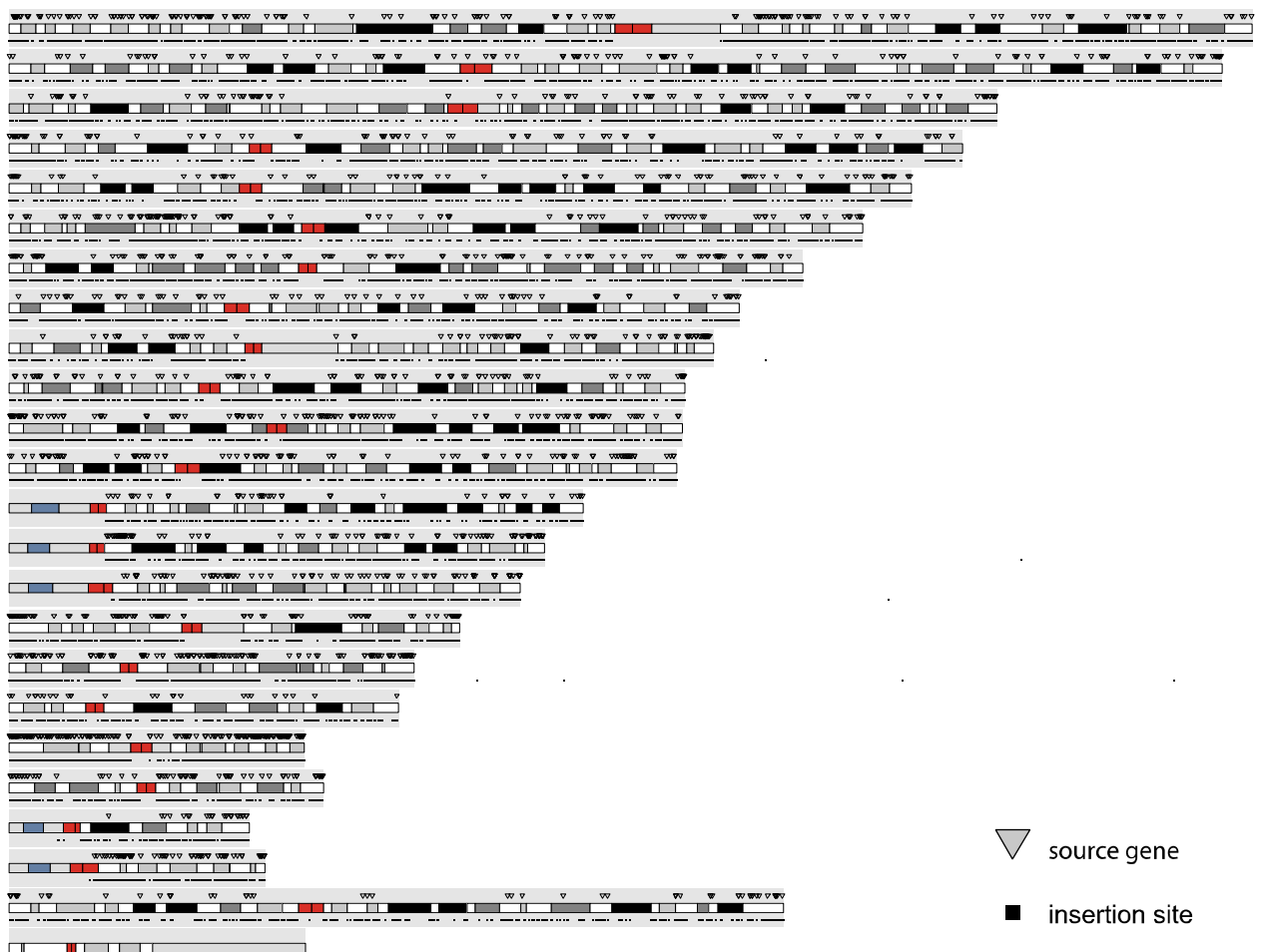

Figure 54. Genomic location of RTs (source gene and insertion locus) detected in gnomAD-

SV database. 


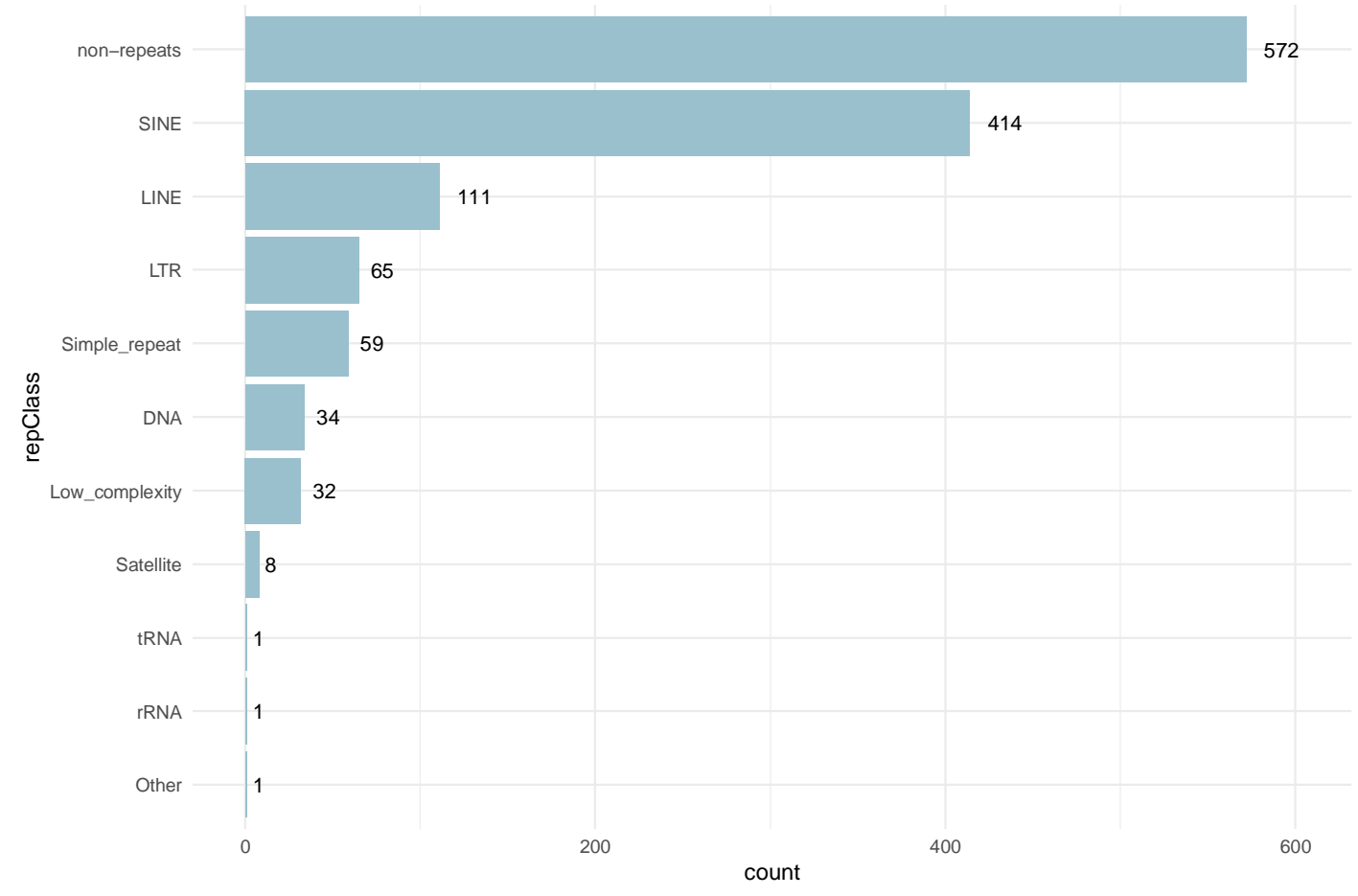

Figure 65. RTs are detectable in gnomAD-SV across different genomic contexts.

RepeatMasker [35] annotations of RT insertion regions.

\section{Conclusion}

We present svaRetro and svaNUMT, R packages developed to facilitate the identification of retro-posed transcript and NUMT insertions. Our tools show outstanding performance on simulation datasets and when benchmarked against existing methods. To further demonstrate its capability, novel RT insertions were discovered by svaRetro on a public population SV database. Integrated into the Bioconductor framework, the packages are compatible with many other available tools for more comprehensive downstream analyses.

\section{Availability of source code and requirements}

- Project name: svaRetro and svaNUMT

- Project home page: 
- svaRetro: https://github.com/PapenfussLab/svaRetro

- $\quad$ svaNUMT: https://github.com/PapenfussLab/svaNUMT

- Operating systems: Platform independent

- Programming language: $\mathrm{R}$

- Other requirements: $\mathrm{R}^{3} 4.1$, Bioconductor ${ }^{3} 3.14$

- License: GPL-3

- RRID:

- svaRetro: SCR_021380

- $\quad$ svaNUMT: SCR_021381

\section{Availability of supporting data and materials}

Data supporting the results of this article are available via the GigaScience repository, GigaDB link TBA.

\section{Declarations}

None declared.

\section{List of abbreviations}

SV: structural variant

NUMT: nuclear mitochondrial integration

RT: retroposed transcript

TSD: target site duplication

mtDNA: mitochondrial DNA 


\section{Ethical approval}

Not applicable.

\section{Consent for publication}

Not applicable.

\section{Competing interests}

The authors declare that they have no competing interests.

\section{Funding}

A.T.P. was supported by an Australian National Health and Medical Research Council (NHMRC) Senior Research Fellowship (1116955) and the Lorenzo and Pamela Galli Charitable Trust. A.T.P and D.C. were supported by an NHMRC Ideas Grant (1188098). J.B. and A.T.P were supported by the Stafford Fox Medical Research Foundation. The research benefitted by support from the Victorian State Government Operational Infrastructure Support and Australian Government NHMRC Independent Research Institute Infrastructure Support.

\section{Author contributions}

A.T.P. conceived the study. R.D. developed the software, wrote the initial draft of the manuscript. D.C. contributed to the software design. J.B. and A.T.P. oversaw the project. All authors reviewed, contributed to, and approved the manuscript.

\section{References}

1. Malhotra A, Lindberg M, Faust GG, Leibowitz ML, Clark RA, Layer RM, et al. 
Breakpoint profiling of 64 cancer genomes reveals numerous complex rearrangements spawned by homology-independent mechanisms. Genome Res. 2013 May;23(5):762-

76.

2. Mahmoud M, Gobet N, Cruz-Dávalos DI, Mounier N, Dessimoz C, Sedlazeck FJ.

Structural variant calling: The long and the short of it. Genome Biology. 2019.

3. MacDonald JR, Ziman R, Yuen RKC, Feuk L, Scherer SW. The Database of Genomic Variants: a curated collection of structural variation in the human genome. Nucleic Acids Res. 2013;42(D1):D986--D992.

4. Sudmant PH, Rausch T, Gardner EJ, Handsaker RE, Abyzov A, Huddleston J, et al. An integrated map of structural variation in 2,504 human genomes. Nature. 2015 Oct;526(7571):75-81.

5. Cameron DL, Schröder J, Penington JS, Do H, Molania R, Dobrovic A, et al.

GRIDSS: sensitive and specific genomic rearrangement detection using positional de Bruijn graph assembly. Genome Res. 2017 Dec;27(12):2050-60.

6. Chen X, Schulz-Trieglaff O, Shaw R, Barnes B, Schlesinger F, Källberg M, et al. Manta: rapid detection of structural variants and indels for germline and cancer sequencing applications. Bioinformatics. 2015;32(8):1220-2.

7. Layer RM, Chiang C, Quinlan AR, Hall IM. LUMPY: a probabilistic framework for structural variant discovery. Genome Biol. 2014;15(6):R84.

8. Cortés-Ciriano I, Lee JJK, Xi R, Jain D, Jung YL, Yang L, et al. Comprehensive analysis of chromothripsis in 2,658 human cancers using whole-genome sequencing. Nat Genet. 2020;

9. Baca SC, Prandi D, Lawrence MS, Mosquera JM, Romanel A, Drier Y, et al. Punctuated evolution of prostate cancer genomes. Cell. 2013;

10. Dayama G, Emery SB, Kidd JM, Mills RE. The genomic landscape of polymorphic 
human nuclear mitochondrial insertions. Nucleic Acids Res. 2014 Nov;42(20):12640-

9.

11. Eiyama A, Kondo-Okamoto N, Okamoto K. Mitochondrial degradation during starvation is selective and temporally distinct from bulk autophagy in yeast. FEBS Lett. 2013 Jun;587(12):1787-92.

12. Higgins GC, Coughlan MT. Mitochondrial dysfunction and mitophagy: the beginning and end to diabetic nephropathy? Br J Pharmacol. 2014 Apr;171(8):1917-42.

13. Ju YS, Tubio JMC, Mifsud W, Fu B, Davies HR, Ramakrishna M, et al. Corrigendum: Frequent somatic transfer of mitochondrial DNA into the nuclear genome of human cancer cells. Genome Res. 2016 May;26(5):717.2.

14. Yuan Y, Ju YS, Kim Y, Li J, Wang Y, Yoon CJ, et al. Comprehensive molecular characterization of mitochondrial genomes in human cancers. Nat Genet. 2020;

15. Puertas MJ, González-Sánchez M. Insertions of mitochondrial dna into the nucleuseffects and role in cell evolution. Genome. 2020;

16. Cooke SL, Shlien A, Marshall J, Pipinikas CP, Martincorena I, Tubio JMC, et al. Processed pseudogenes acquired somatically during cancer development. Nat Commun. 2014 Apr;5:3644.

17. Gherman A, Chen PE, Teslovich TM, Stankiewicz P, Withers M, Kashuk CS, et al. Population bottlenecks as a potential major shaping force of human genome architecture. PLoS Genet. 2007 Jul;3(7):e119.

18. Han YJ, Ma SF, Yourek G, Park Y-D, Garcia JGN. A transcribed pseudogene of MYLK promotes cell proliferation. FASEB J. 2011 Jul;25(7):2305-12.

19. Hawkins PG, Morris K V. Transcriptional regulation of Oct4 by a long non-coding RNA antisense to Oct4-pseudogene 5. Transcription. 2010 Nov;1(3):165-75.

20. Poliseno L, Salmena L, Zhang J, Carver B, Haveman WJ, Pandolfi PP. A coding- 
independent function of gene and pseudogene mRNAs regulates tumour biology.

Nature. 2010 Jun;465(7301):1033-8.

21. Obenchain V, Lawrence M, Carey V, Gogarten S, Shannon P, Morgan M.

VariantAnnotation: A Bioconductor package for exploration and annotation of genetic variants. Bioinformatics. 2014;

22. Cameron D, Dong R. StructuralVariantAnnotation: Variant annotations for structural variants. [Internet]. 2020. Available from:

https://bioconductor.org/packages/release/bioc/html/StructuralVariantAnnotation.html

23. Lawrence M, Huber W, Pagès H, Aboyoun P, Carlson M, Gentleman R, et al.

Software for Computing and Annotating Genomic Ranges. PLoS Comput Biol. 2013;

24. Lawrence M, Gentleman R, Carey V. rtracklayer: An R package for interfacing with genome browsers. Bioinformatics. 2009;

25. Quinlan AR, Hall IM. BEDTools: A flexible suite of utilities for comparing genomic features. Bioinformatics. 2010;

26. Craig DW, Nasser S, Corbett R, Chan SK, Murray L, Legendre C, et al. A somatic reference standard for cancer genome sequencing. Sci Rep. 2016;

27. Zook JM, Hansen NF, Olson ND, Chapman L, Mullikin JC, Xiao C, et al. A robust benchmark for detection of germline large deletions and insertions. Nat Biotechnol. 2020 Jun 15;

28. Huang W, Li L, Myers JR, Marth GT. ART: A next-generation sequencing read simulator. Bioinformatics. 2012;

29. Ewing AD, Ballinger TJ, Earl D, Sequencing BIG, Program A, Platform, et al. Retrotransposition of gene transcripts leads to structural variation in mammalian genomes. Genome Biol. 2013 Mar;14(3):R22.

30. Zook JM, Catoe D, McDaniel J, Vang L, Spies N, Sidow A, et al. Extensive 
sequencing of seven human genomes to characterize benchmark reference materials.

Sci Data. 2016;

31. Collins RL, Brand H, Karczewski KJ, Zhao X, Alföldi J, Khera A V., et al. An open resource of structural variation for medical and population genetics. bioRxiv [Internet]. 2019;(October):doi: 10.1101/578674. Available from:

http://dx.doi.org/10.1101/578674

32. Smit A, Hubley R, Green P. RepeatMasker Open-4.0. 2013-2015 .

http://www.repeatmasker.org. 2013. 